\title{
Telemetry System Based on MESH Network and Its Application
}

\author{
Pingfan Guo, Ming Liu, Hong Li, Hongxiang Zhu \\ (Chinese Flight Test Establishment, Xi'an, 710089, China) \\ liu130999@163.com
}

\begin{abstract}
In the flight test, the advantages of network telemetry have gradually emerged, and their application fields will also be expanded. This paper introduces a network telemetry system based on MESH net and its application in flight test, a ground station can receive telemetry signals of several planes at the same time; the components and functions of the system are described, the advantages of this network telemetry system, existing problems and suggestions on future improvements are presented.
\end{abstract}

\section{INTRODUCTION}

Telemetry monitoring technology is a comprehensive technology. It integrates sensing, acquisition, communication and data processing and is widely used in flight test. At present, since the aviation industry is booming, current mainstream S-band telemetry system based on the PCM telemetry architecture is facing with shortage of resources at frequency points, telemetry bandwidth requirement become an increasingly wider, and its work mode is not flexible due to one frequency point for an airplane. With the increasing the number of flight test aircrafts and the expansion of the telemetry data bandwidth, it is difficult to meet the new challenge. At the same time, the PCM-based telemetry system architecture can only achieve point-to-point transmission, and each ground receiving station antenna can only track one aircraft which is not flexible.

The PCM-based telemetry system architecture cannot meet the needs of multi-aircraft, highintensity, and high-efficiency missions in aspects of guaranteeing flight inspection efficiency and safeguarding capabilities. With the expansion and improvement of the IRIG106 standard, network telemetry technology begin to be gradually generated and applied. Combined with wireless MESH network technology, a wireless MESH network telemetry system has become a newer and better choice for telemetry systems. This paper designs a wireless telemetry system based on wireless MESH which has been applied in the flight test telemetry system.

\section{MESH network technology}

\subsection{MESH Network Technology Overview}

The wireless MESH network is a newly emerged wireless multi-hop mesh topology network. It can be combined with multiple broadband wireless access technologies to form a wireless mesh network with multi-hop wireless links. This kind of wireless mesh network is a kind of "multi-hop" network [1]. Any wireless device node can act as both an AP and a router. Each node in the network can send or receive signals and directly communicate with one or more peer nodes. In this way, the coverage of wireless systems can be greatly increased, while the bandwidth capacity and communication reliability of wireless systems is improved. It is an ideal solution for the expansion of the flight test telemetry system. 


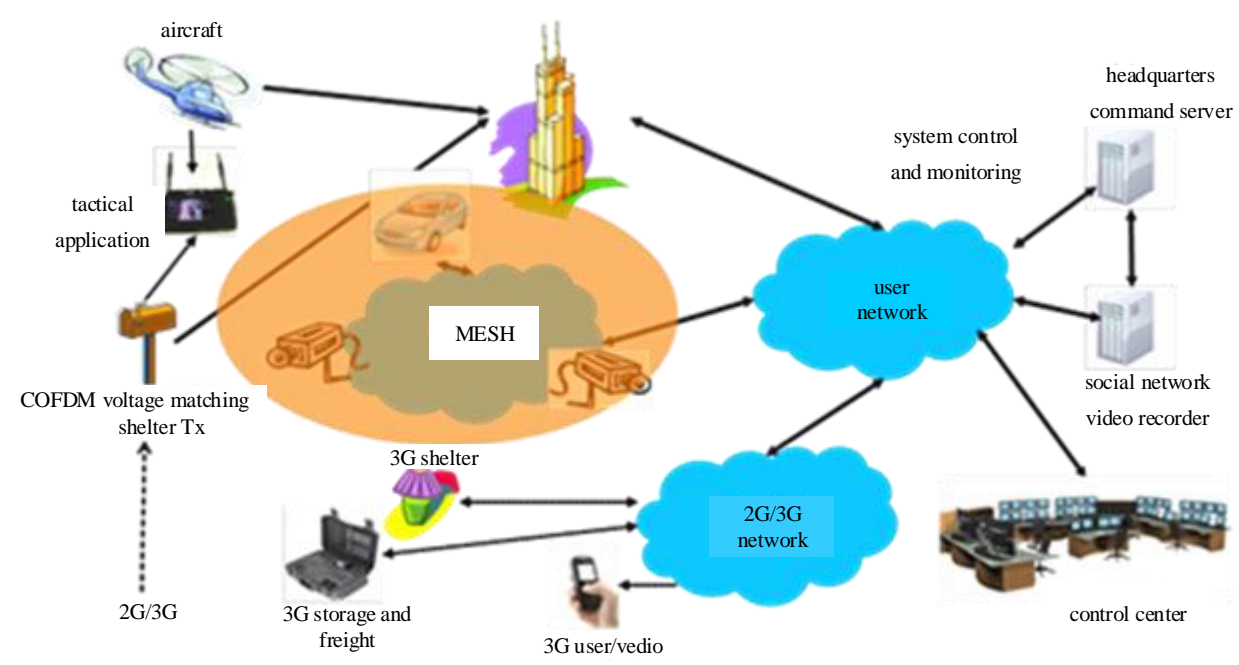

Fig. 1 Typical Application of MESH Network. [2]

\subsection{Features of Wireless MESH Network}

Wireless MESH network has features such as flexible network structure, easy deployment and configuration, fault tolerance, and multipoint-to-multipoint communication with mesh connections, which makes the initial deployment cost of the wireless MESH network very low, and can be expanded according to demand. The self-organizing and self-healing capability enables the wireless MESH network to eliminate the need for an administrator to manually configure the network, automatically discover new nodes, automate the configuration process, automatically maintain the network's normal operation, and automatically adjust the network for self-healing when node/link failures occur. [3]

The specific features are as follows:

- Self-configuration: with automatic configuration and centralized management capabilities to simplify the management and maintenance of the network;

- Self-healing: Provides redundant paths with automatic discovery and dynamic routing to eliminate single point of failure effects on the system;

- Wide bandwidth: Unlike traditional wireless LANs, network bandwidth does not decrease with distance;

- Redundancy and scalability: Each node has one or more paths for data transmission. The number of nodes can be expanded to hundreds or even thousands.

\section{Wireless Telemetry System Based on Wireless MESH}

The wireless MESH-based network telemetry system designed on the basis of the above characteristics mainly includes an on-board test system and a ground control/receiving system with the two-way communication telemetry link. This system can solve the increasingly huge challenge faced by the existing PCM architecture telemetry system.

The airborne test system collect outputs network data to the network transceiver. The transceiver modulates the signal and transmits it. At the same time, it receives control signals from the ground to implement link initialization and IP control functions. The network telemetry transmission subsystem is mainly used for downlinking the network data flow output by the airborne test unit and uploading ground control commands to control the airborne test equipment. The ground antenna is an omnidirectional antenna, which can receive the data of the airborne network of three aircrafts 
at the same time, and uplink data for the link control.

The airborne acquisition and recording subsystem mainly completes the extraction and collection of airborne video signals and airborne 429, 422 bus data, and encodes the acquired video and key parameters into network data packets, then transmits them from the airborne Internet radio station of the network telemetry sub-system to the ground omnidirectional antenna of telemetry receiving subsystem. The terrestrial network radio transfers the received network data packet to the ground telemetry security monitoring server through the Ethernet switch. The server sends the real-time parameters of the aircraft to the monitoring client through the network multicast to realize real-time ground monitoring and quasi-real-time flight performance analysis.

At the same time, the airborne acquisition and recording subsystem can receive the uplink data command from the ground control center, complete the device self-test, self-start, restart, downlink data bit rate settings and other operations. The GPS/Beidou integrated positioning device with realtime differential and timed functions can be installed to provide accurate positioning measurement of the trajectory and speed of the aircraft. The overall design of a telemetry system based on a wireless MESH network is shown in Figure 2.

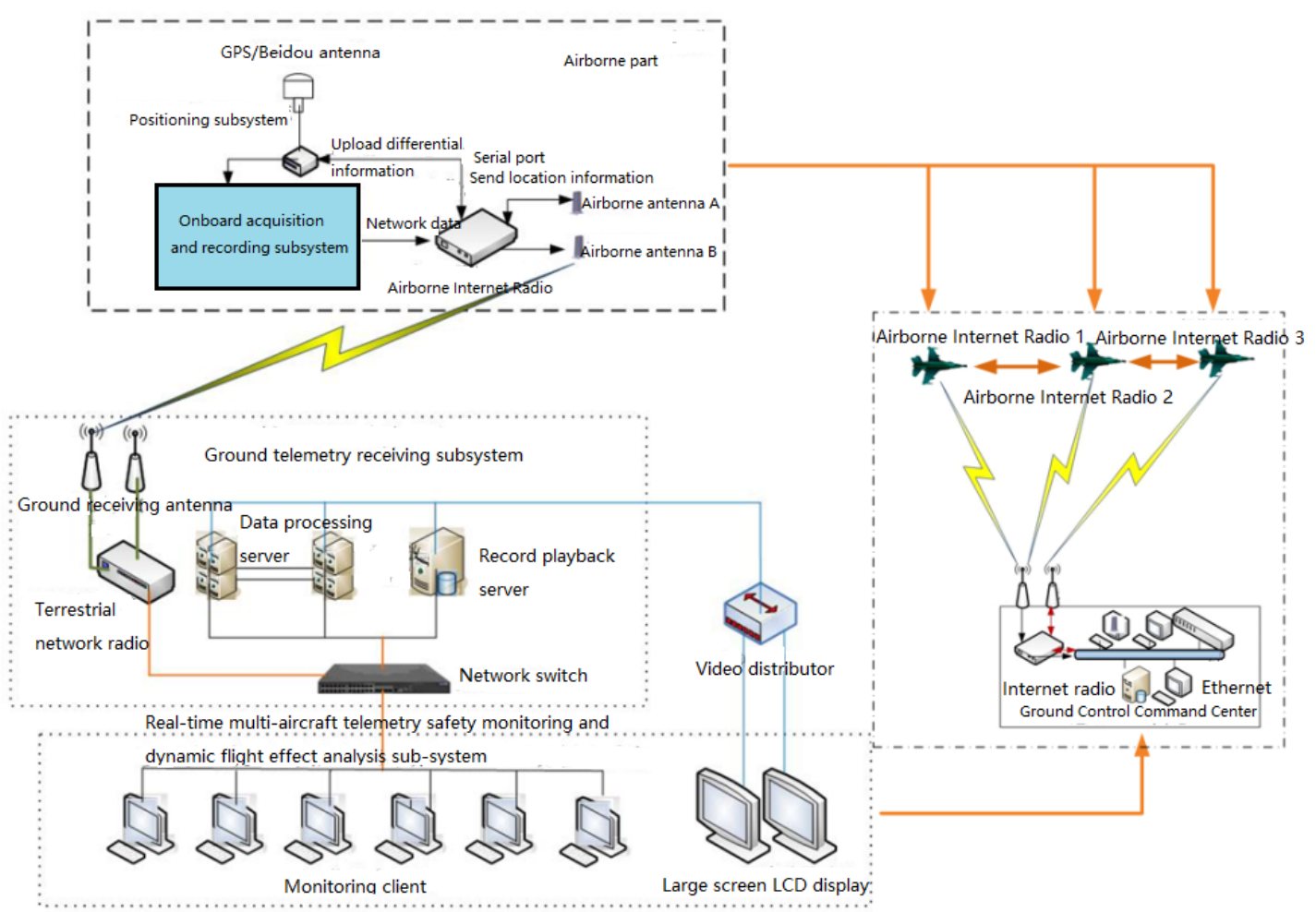

Fig. 2 Block diagram of telemetry system based on wireless MESH network.

Main Functions of the telemetry system based on wireless MESH network:

a. Up to 16 IP wireless nodes to form a "non-fixed self-healing wireless network";

b. Telemetry from one point to multipoint;

c. The wireless network works in a narrow band at the same frequency (optional bandwidth 2.5, $3.0,3.5,5.0,6.0 \mathrm{MHz})$;

d. The system uses COFDM to achieve excellent transmission range and beyond-line-of-sight transmission capability;

e. The wireless network is multidirectional;

f. Each node can be used as an input source for video, audio or IP data; 
g. Any node can act as a relay point;

h. Transmission mode is TCP/IP, UDP transparent transmission;

i. Anti-multipath fading and Doppler shift mechanisms.

The network telemetry system based on MESH has been applied in flight test. One set of terrestrial receiving base stations in the system monitors and measures transmitted video signals of no less than 3 aircrafts, and monitors and measures the number of aircrafts not less than 5 when no video is transmitted. The cross-link state diagram of the airborne network telemetry transmitter of the three aircrafts and two terrestrial network telemetry receivers is shown in Figure 3.

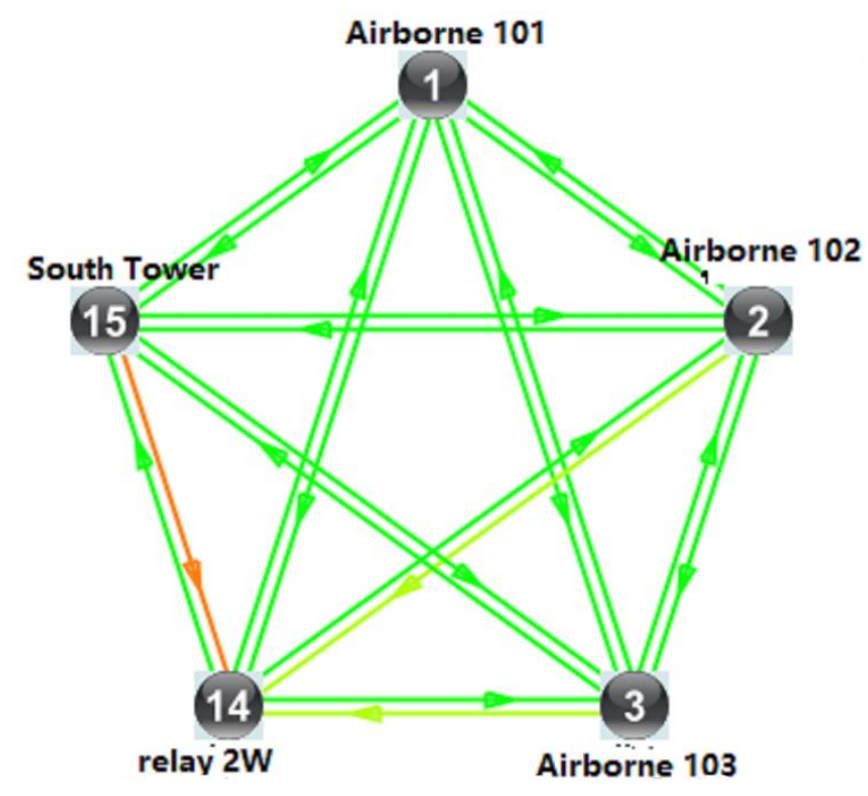

Fig. 3 Multi-system cross-link state diagram.

\subsection{Onboard subsystem design}

The airborne network sub-system can combine a self-organizing and self-healing wireless mesh network at most consisting of 16 nodes. Nodes within the mesh network can exchange data at the same frequency, simplifying frequency management. This mesh only occupies $2.5 \mathrm{MHz}$ bandwidth $(3.0,3.5,5.0$, and $6.0 \mathrm{MHz}$ are also available). The node adopts a unique COFDM modulation mechanism. Therefore, it has excellent radio frequency penetrating ability and multi-path transmission performance.

Wireless mesh node can provide IP data transmission rate of more than $8.0 \mathrm{Mb} / \mathrm{s}$ (The rate depends on the model of the node, the number of nodes, and the distance between nodes).

This IP rate is used to exchange IP service data between nodes. A highly flexible mesh structure means that data between nodes can be exchanged point-to-point or point-to-multipoint; nodes can also increase transmission distance through relay transmissions. Airborne network subsystem architecture as shown in Figure 4. 


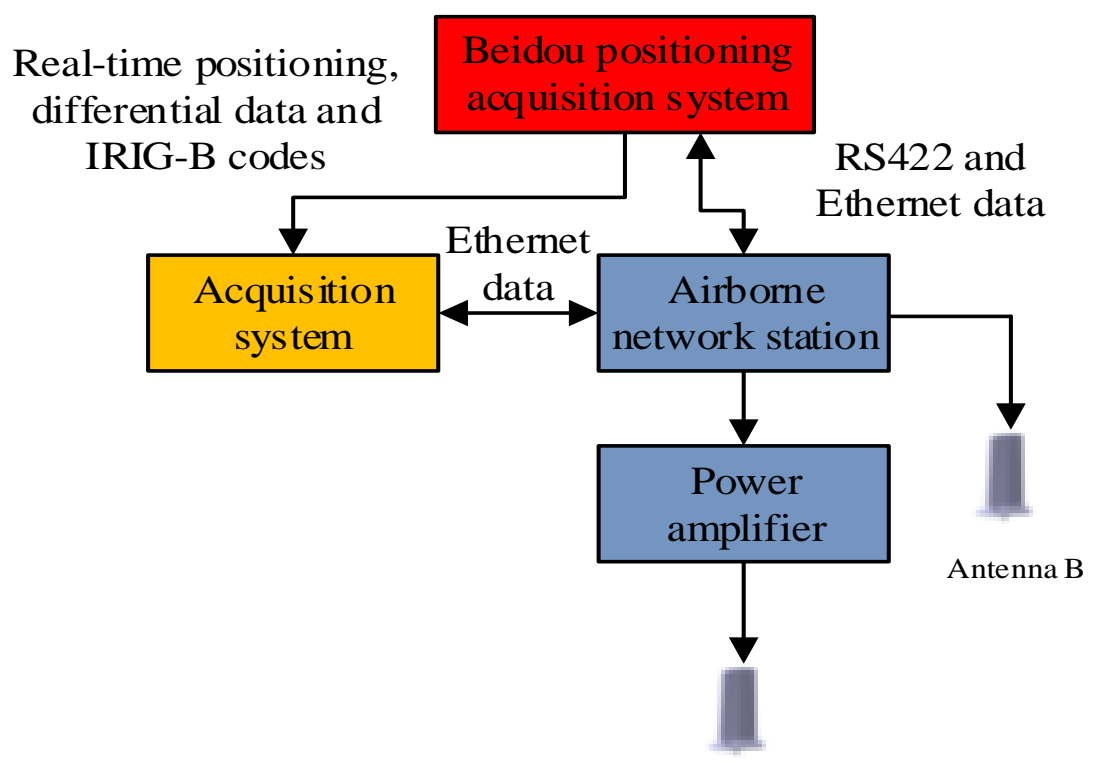

Antenna A

Fig. 4 Airborne Network Subsystem Architecture.

\subsection{Ground receiving subsystem design}

The ground telemetry receiving subsystem receives the airborne telemetry transmission data, processes it in real time, and sends it to the ground control command center to display the key realtime parameters of the aircraft and real-time/quasi-real-time pilot flight effect analysis through the optical fiber network. At the same time, uplink differential navigation data is transmitted to the aircraft for real-time differential position of GPS/Beidou receiver. It solves the issue of the lack of real-time position monitoring accuracy and post-distribution differential processing on the ground after the completion of the previous flight and reduces data processing time by at least 2 Hours. CFTE achieved real-time dynamic reference differentials through years of technical research.

The ground telemetry receiving subsystem includes three parts: ground telemetry receiving subsystem, ground real-time differential reference subsystem, and ground telemetry control subsystem. The system is mainly composed of two sets of omnidirectional telemetry receiving antennas, network connection/transmission modules, power amplifiers, real-time processing units, etc. One set of receiving system can remotely measure the data of three aircrafts. The ground telemetry receiving subsystem is used for receiving and demodulating the telemetry data, and the terrestrial real-time differential reference subsystem is used to upload the reference station data through the wireless radio station for real-time differential, thereby obtaining high-precision realtime positioning information. The ground real-time differential subsystem can also be used to solve high-precision user trajectory data based on GPS/Beidou original data recorded by ground differential reference stations and airborne GPS/Beidou positioning equipment for analysis of aircraft flight trajectory data and other related analysis and processing.

The ground telemetry control subsystem mainly completes the work state monitoring and equipment parameter configuration of on-board acquisition and recording equipment for terrestrial network radio stations, multi-aircraft airborne internet radio stations and multiple aircrafts, including two sets of terrestrial telemetry control computers and ground telemetry control software. The ground telemetry control computer uses the server of the ground telemetry receiving subsystem to control the equipment and the link. Ground control command center equipment connection diagram shown 
in Figure 5.

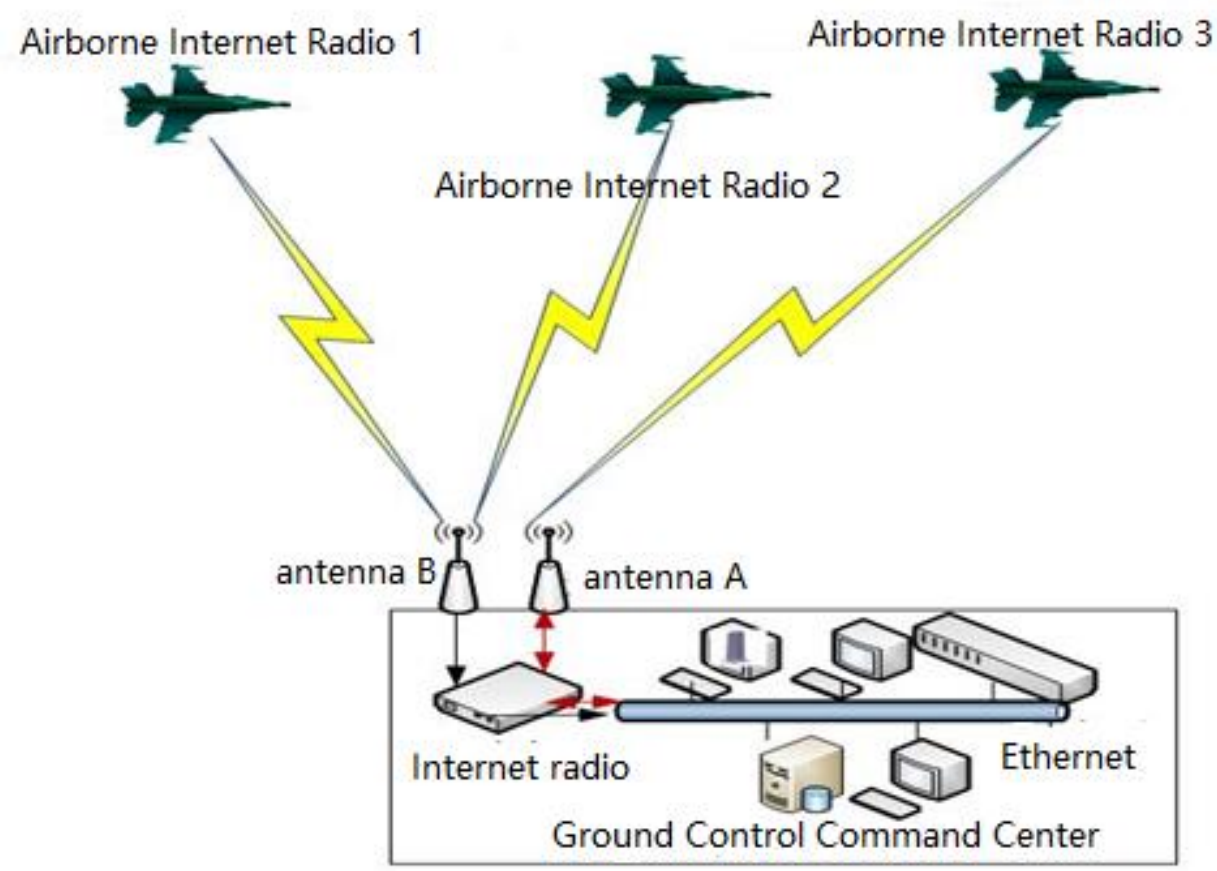

Fig. 5 ground control command center equipment connection diagram.

\section{Network telemetry system data packet structure design}

The new network telemetry system must satisfy both the existing IRIG 106 telemetry standards and the published iNET telemetry network standards to achieve both new functions and compatibility with existing systems. For the above principles, the wireless mesh network in this paper is designed as a wireless network transparent to Ethernet. Ethernet packets can be transparently transmitted without any changes. Transparent transmission is achieved through re-encapsulation of Ethernet data packets. Ethernet data packets are re-encapsulated with reference to the IEEE 802.11 standard. The format after the package is shown in Figure 6[4].

Network header 1: Identify the sending and receiving addresses of two nodes that send and receive wireless single-hop. In the transmission process, every time a node passes through, the header 1 will change: the node that receives the packet will tear down the header 1 , and when it is sent out, it repackages the new header and changes the address of the sending node to the current sending node. Address, change the address of the receiving node to the address of the next hop receiving node.

Network header 2: Identifies the destination node and source node address of the packet. In the data packet transmission process, the packet header 2 is not changed. Only when the packet reaches the real destination node will the packet header be removed to obtain the destination address of the Ethernet frame and forwarded to the corresponding destination host. Ethernet data packets generated by the user host are encapsulated by the access node in the above format and transmitted in the wireless mesh network. The routing node queries the forwarding table according to the destination address of the data packet to obtain the next hop address of the data packet, and forwards the data packet to the next hop node.

\begin{tabular}{|l|c|c|c|c|c|c|c|}
\hline receiving node & sending node & destination node & source node & destination MAC & source MAC & type & data \\
\hline
\end{tabular}

Fig. 6 Network Telemetry Packet Structure. 


\section{Network Telemetry System Network Management Technology}

According to the two-way communication function of the telemetry link in the wireless MESH network telemetry system, and the MESH network supports the SNMP (Simple Network Manage Protocol). The test network system (agNET) management technology is designed and developed for the telemetry system in MDL (Metadata Description Language) to solve test and experiment network system architecture, requirements, mission description and management taskes. For the requirements of flight test and test network system management, referring to related standards such as system management standard (SM) and metadata standard (MD) in iNET, with SNMP protocol the test network system management is developed in MDL language. The software implements the three major management functions for system configuration, system status and system security through informational means and friendly human-machine interface to ensure stable, orderly and efficient operation of the flight test and test network system.

The MDL-based flight test and test network system management technology is based on the standard SNMP protocol and the specific requirements of the flight test and test network system, The system structure, function, requirement description and configuration are implemented through the MDL to reduce the different of each components, with the MESH network telemetry two-way link to achieve the three major management functions of the test object, ground station, telemetry network, etc. in the test and test network can meet the requirements for dynamic configuration, performance monitoring, fault location, user authentication, and data encryption for test and test networks in tests, improve system service quality, and ensure optimal performance and stable operation of test and test network systems. The management activities run through the entire process of system operation and cover all parts of the system. The network system management unit is the nerve center of the entire test and test network system and becomes one of the core parts of the entire system. Lack of management, network, will become inefficient, disorderly, uncontrollable, and eventually endanger. Network system management technology is a key technology in the entire MESH network telemetry system.

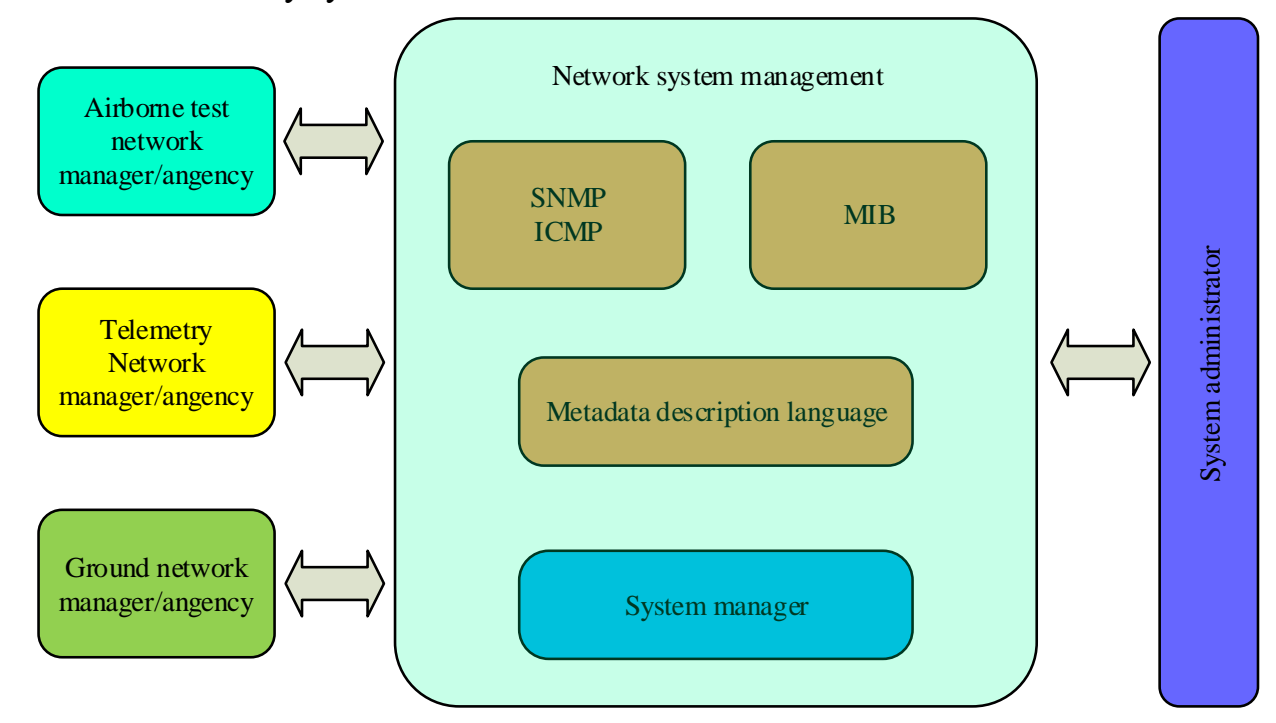

Fig. 7 Schematic diagram of network management based on MESH network telemetry system.

\section{CONCLUSIONS}

The wireless MESH network-based telemetry system implements a network-based single-frequency 
multi-target telemetry technology, satisfies the multi-target monitoring flight test requirements, breaks through the limitations of traditional telemetry systems, and promotes the development of new telemetry technologies.

The wireless telemetry solution based on wireless MESH is a hybrid of multiple new technologies such as MESH, COFDM, and 16QAM. It can complement each other in the current telemetry system and is a cost-effective telemetry system capable of meeting various tasks (especially small and medium scale) demand, and Unattended system maintenance can be achieved to achieve pointto-multipoint telemetry monitoring. However, due to the complexity of wireless MESH network links, multiple delays in data forwarding may cause high delays, and real-time performance cannot be guaranteed in video telemetry. These deficiencies need further study. Combined with the development of new technologies, such as MIMO technology, the system will enable higher bandwidth for telemetry data in the future, thus broadening its application fields.

\section{REFERENCES}

[1] Antonio Montejo; Alberto Bañacia; Hirokazu Sawada; Kentaro Ishizu; Kazuo Ibuka; Fumihide Kojima, "Implementation of a multi-hop network at the university campus using an IEEE 802.11af-compliant Network," 2017 20th International Symposium on Wireless Personal Multimedia Communications (WPMC), pp. 173-180, 17-20 Dec. 2017.

[2] Rohith Unnikrishnan; S. Renuga Devi; Rachita Ramesh; A. Rajesh; Aditya Varma, "Investigation on fast recovery congestion control algorithms for IEEE 802.11e based wireless mesh networks," 2017 International Conference on Inventive Computing and Informatics (ICICI), pp. 217 - 221, 23-24 Nov. 2017. 2017.

[3] Yuanbo Cai and Jingjing Zheng, Application technology of wireless Mesh network. Publishing House of Electronics Industry: Beijing, 2015.

[4] IRIG 106 Chapter 23 Draft 01 Release; Range Commanders Council U.S. Army White Sands Missile Range New Mexico 88002-5110. 